\title{
VHF/UHF Kapton supported antenna for cubesat applications
}

\author{
A. Takacs $^{1}$, S. Charlot ${ }^{1}$, P. Calmon ${ }^{1}$, D. Dragomirescu ${ }^{1}$ \\ LAAS-CNRS, Université de Toulouse, CNRS, INSA, UPS Toulouse, France \\ atakacs@laas.fr, charlot@laas.fr, calmon.fr, daniela@laas.fr
}

\begin{abstract}
This paper addresses an original concept for very low mass planar flexible Kapton supported antenna for cubesat application. In order to illustrate the concept a dual-band VHF/UHF planar cross dipole antenna is presented. This circularly polarized antenna is compatible with the integration requirements for a cubesat mission and demonstrates very good performances in terms of input matching and radiation pattern.
\end{abstract}

Keywords - dual-band, VHF/UHF antenna, cubesat, flexible planar antenna

\section{INTRODUCTION}

The interest for Cubesat (CS) miniaturized satellite for space research program rising exponentially in last years. Their small size, ranging from $0.5 \mathrm{U}$ to $6 \mathrm{U}(1 \mathrm{U}$ is a volume of $10 \mathrm{~cm}$ x $10 \mathrm{~cm} \mathrm{x} 11 \mathrm{~cm}$ ) and low weight (typically less than $10 \mathrm{~kg}$ for a $6 \mathrm{U} C S$ ) involve very challenging design requirements mainly for DC power units (including battery and solar cells) and RF units (including transceivers and antennas). Antennas are used for TTC (Telemetry, Tracking and Control) functions or (scientific) data communications. The TTC function requires a very reliable low bit rate radio link established between the CS and the ground station (GS). This radio link should be effective even in a worst case scenario (e.g. when the DC power reserve of the CS is very low or when the satellite orientation with the respect of GS is not well controlled). Thus the VHF or/and UHF frequency bands are privileged because of the 'low' propagation losses between the GS and CS (as compared with higher frequency bands). But VHF and even UHF antennas are large and bulky for CS and Hold-Down and Release Mechanism (HDRM) should be used in order to fit the antenna in the allowed CS volume during launching and then deploying them during the flight mission. Typically wire antennas (e.g. dipole, monopole, turnstile, helical, quadrifilar helix) are used for such TTC functions in VHF/UHF bands [1]. For the first time, we present here a novel concept of self-deployable planar VHF/UHF antennas based of the use of a flexible kapton technology. The design of the proposed kapton supported antennas is presented in Section II while the simulation results are presented in Section III.

\section{Kapton Supported Cross Dipoles ANTENNA}

Kapton is a polyimide based material combining very good mechanical, thermal, chemical and electrical properties [2]. Kapton is already used for space various space instruments embarqued on spacecraft and satellites. The electrical properties of the Kapton (dielectric constant: 3.4, loss tangent: 0.0018 ) recommended Kapton as a good candidate for flexible antennas [3]-[4]. Kapton HN film is available with thickness

of: $25 \mu \mathrm{m}, 50 \mu \mathrm{m}, 75 \mu \mathrm{m}$ and $125 \mu \mathrm{m}$ and can be used as support for very thin planar and flexible antennas. The Kapton mass is only $3.55 \mathrm{mg} / \mathrm{cm} 2$ (thickness $25 \mu \mathrm{m}$, no metallization) and $5.1 \mathrm{mg} / \mathrm{cm} 2$ (thickness $25 \mu \mathrm{m}$ with $0.8 \mu \mathrm{m}$ of gold metallization). A VHF Planar Cross Dipole Antenna (PDCA) operating at $144 \mathrm{MHz}$ (assuring the TTC function) was designed and simulated in order to illustrate the proposed concept (VHF kapton antenna for CS applications). This is a 'worst case' design scenario because at such low frequencies the antenna is typically large and bulky. The proposed PCDA is composed by two half-wavelength planar dipoles disposed in cross configuration as shown in Fig. 1 a) and fed in phase quadrature. A review of crossed dipole antennas is presented in [5]. Elliptical holes are performed into the dipoles in order to reduce both the mass and the aerodynamic footprint of the antenna. Before the deployment the antenna should be folded as illustrated in Fig. 1 b). A HDRM based on split spool device/thermal knife is intended to be used. We note that no spring based mechanism is required for PCDA as for the 'classical' VHF wire antennas. The tensile stress of the flexible kapton film (that can be controlled and tuned by an appropriate technological process [4]) is enough to deploy the antenna once the hold-down mechanism released.

a)

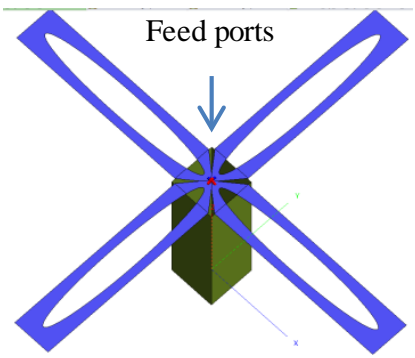

Fig. 1. Proposed kapton supported PCDA : a) deployed on the $3 U$ CS, and b) folded before deployment (CS body is not represented)

The main dimensions of the PDCA are reported in the Fig. 2. The length ' $\mathrm{L}$ ' should be in the range of the quarter wavelength and basically fix the operating frequencies. The width ' w' should be accomodated with the mechanical design of the CS ( $<10 \mathrm{~cm}$ for a $3 \mathrm{U} \mathrm{CS})$. This dimension can be larger with the benefit of the increased frequency bandwith of the PCDA but with the expense of a more complicated folding procedure and HDRM. Because the electrical currents flows mainly on the exterior contour of the PCDA a hole can be introduced in order to reduce the weight and the aerodinamic footprint of the antenna. For simplicity reasons an elliptical hole was used. The kapton substrate is keep only under the metallized region (blue in Fig. 2). 


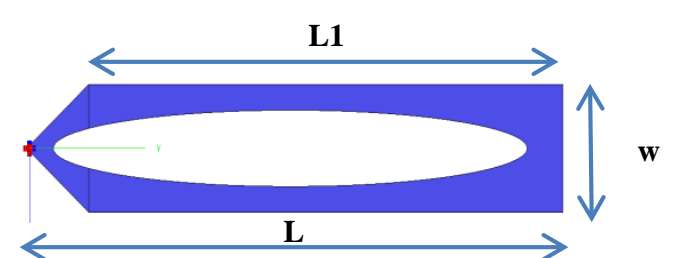

Fig. 2. Top view of the half of each constituve dipole of the PCDA with its main dimmensions

\section{RESULT}

Full-wave electromagnetic simulations were performed by using Feko software (Method of Moment). The body of the CS was modelled as a lossy metallic cuboid (Al based) of $10 \mathrm{~cm} \mathrm{x}$ $10 \mathrm{~cm} \times 34 \mathrm{~cm}$ and was taken into account in the simulation. First, a parametric analysis was performed in order to quantify the impact of the main dimensions of the PCDA: length (L) and width (w). The length was keep constant $\mathrm{L}=45.5 \mathrm{~cm}$ $(\mathrm{L} 1=41 \mathrm{~cm})$ while $\mathrm{w}$ was varied from $2 \mathrm{~mm}$ to $84 \mathrm{~mm}$. No hole was considered for this analysis. The simulated reflection coefficient is represented in Fig. 3 while the maximum gain is depicted in Fig. 4. As shown in Fig. 3 the PCDA exhibits two resonant frequencies (targeted $144 \mathrm{MHz}$ and $432 \mathrm{MHz}$ ) corresponding to $\lambda / 2$ and $3 \lambda / 4$ operating mode. The resonant frequencies are increased while $\mathrm{w}$ decreased and the frequency bandwidth is increased while $\mathrm{w}$ increases. We note that, as expected, the PCDA exhibits a dual band behavior with $\lambda / 2$ (VHF frequency) and 3N/4 (UHF frequency) radiation pattern. Thus a single PCDA can be used for uplink (e.g. $144 \mathrm{MHz}$ ) and downlink (e.g. VHF) path. As illustrated in Fig. 4 the 'VHF' gain (at $144 \mathrm{MHz}$ ) is not significantly impacted by the width parameter ' $w$ ' while the UHF gain (at $432 \mathrm{MHz}$ ) increased while w decreased.

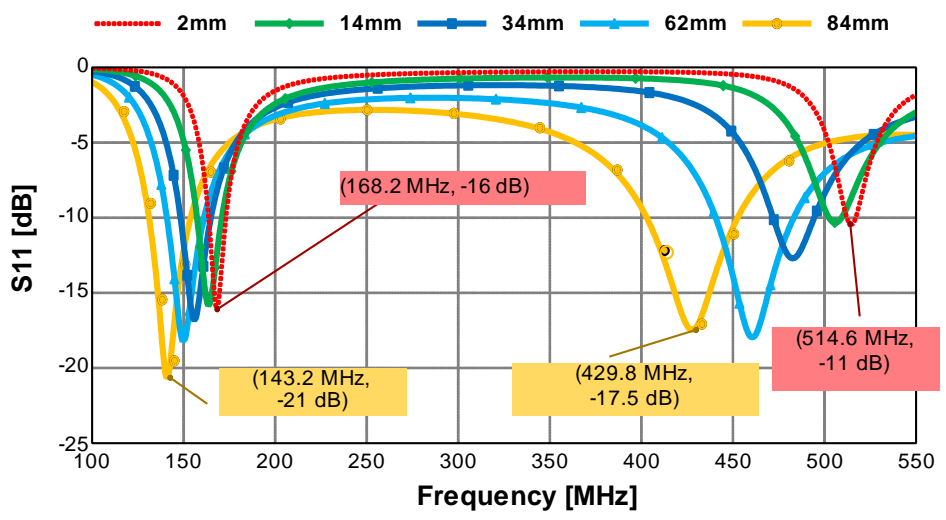

Fig. 3. Reflection coefficient (simulation results, FEKO) for various width 'w' while the length $\mathrm{L}$ is keep constant at $\mathrm{L}=45.5 \mathrm{~cm}$.

A parametric analysis concerning the dimensions of the elliptical hole was also conducted. It was found that the holes dimensions do not impact significantly the VHF resonant frequency and impact moderately the UHF frequency. Finally a PCDA $(\mathrm{L}=45.5 \mathrm{~cm}, \mathrm{~L} 1=41 \mathrm{~cm}, \mathrm{w}=84 \mathrm{~mm})$ with an elliptical hole (minor radius: $2.5 \mathrm{~cm}$ and a major radius $20 \mathrm{~cm}$ ) was selected. The mass of this PCDA design is approximately $3.9 \mathrm{~g}$ (kapton thickness $25 \mu \mathrm{m}$ with $0.8 \mu \mathrm{m}$ of gold metallization). For a no-hole PCDA design the mass is expected to be twice higher. The manufacturing process developed in our laboratory [4] is intended to be used. This process allows metallization quality and manufacturing resolutions far beyond the requirements of VHF/UHF applications but, as a matter of principle, other low-cost process based on inkjet printing can be used. The simulation results (Fig. 5) show very good results in terms of circular polarization (CP) at $144 \mathrm{MHz}$ (the diference between co and cross components of the gain is greater than $10 \mathrm{~dB}$ in an angular bandwith of $104^{\circ}$ ). Good CP behaviour is obtained also at $434 \mathrm{MHz}$ but in a lower angular bandwith because of a typical $3 \lambda / 4$ radiation shape.

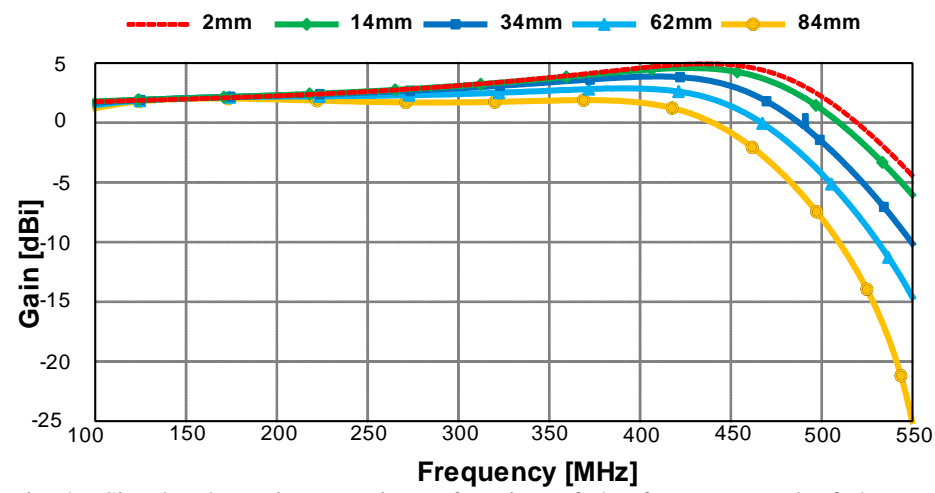

Fig. 4. Simulated maximum gain as function of the frequency and of the width of the PCDA.

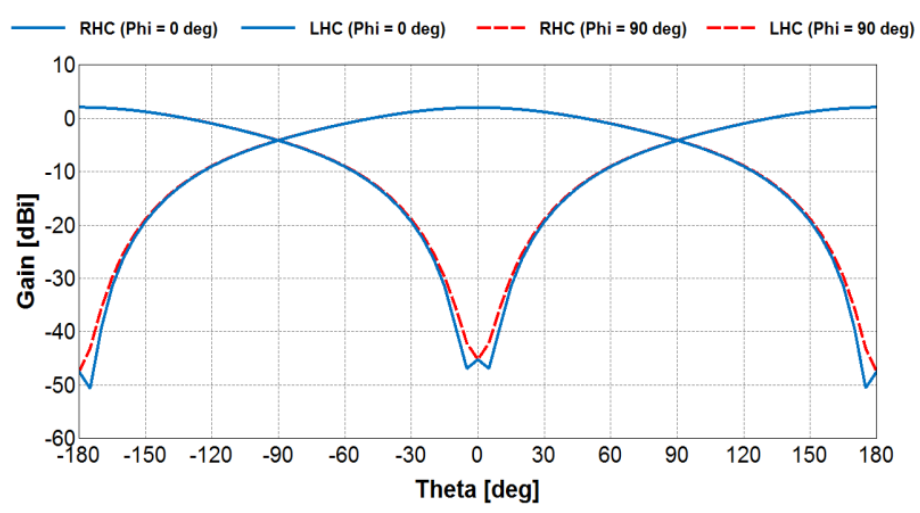

Fig. 5. Co (LHC, continuous blue line) and cross (RHC, dotted red line) components of the gain obtained in $\mathrm{XoZ}\left(\mathrm{phi}=0^{\circ}\right)$ and $\mathrm{YoZ}\left(\mathrm{phi}=90^{\circ}\right)$ cuts. Simulations results obtained at $144 \mathrm{MHz}$ by using FEKO (MoM) software

\section{CONCLUSION}

A dual-band Kapton supported planar crossed dipole antenna for cubesat applications was proposed. This very low weight (mass $<4 \mathrm{~g})$ antenna is easy to integrate into a cubesat as VHF/UHF antenna. The design methodology was presented with an emphasis of the design guidelines. The electromagnetic simulations predict very good performances in terms of input matching and radiation pattern. The manufacturing of such antenna is under run and experimental results will be presented at conference.

\section{REFERENCES}

[1] W. A. Imbriale, S. Gao, L. Boccia "Space Antenna Handbook", John Wiley \& son, 2012.

[2] http://www.dupont.com/content/dam/assets/products-and-services /membranesfilms/assets/DEC-Kapton-summary-of-propert ies.pdf

[3] Z. Yang, A. Takacs, S. Charlot, D. Dragomirescu, "Fle xible Substrate Technology for Millimeter Wave Wireless Power Transmission", Wireless Power Transfer (Cambridge University Press), Vol.3, $\mathrm{N}^{\circ}$, pp.24-33, March 2016

[4] Z. Yang, "Flexible Substrate Technology For Millimeter Wave Applications”, Ph. D. thesis, 19 Dec. 2016.

[5] S. X. Ta; I. Park; R. W. Ziolkowski, "Crossed Dipole Antennas: A review”, IEEE Ant. and Propag. Magazine, Vol. 57, Issue: 5, 2015. 